\title{
Sterile neutrino dark matter and low scale leptogenesis from a charged scalar
}

\author{
Michele Frigerio $^{1}$, Carlos E. Yaguna ${ }^{2, \mathrm{a}}$ \\ ${ }^{1}$ Laboratoire Charles Coulomb, UMR 5221 (CNRS/Université Montpellier 2), 34095 Montpellier, France \\ ${ }^{2}$ Institut für Theoretische Physik, Universität Münster, Wilhelm-Klemm-Straße 9, 48149 Münster, Germany
}

Received: 24 September 2014 / Accepted: 26 December 2014 / Published online: 27 January 2015

(C) The Author(s) 2015. This article is published with open access at Springerlink.com

\begin{abstract}
We show that novel paths to dark matter generation and baryogenesis are open when the standard model is extended with three sterile neutrinos $N_{i}$ and a charged scalar $\delta^{+}$. Specifically, we propose a new production mechanism for the dark matter particle-a multi-keV sterile neutrino, $N_{1}-$ that does not depend on the active-sterile mixing angle and does not rely on a large primordial lepton asymmetry. Instead, $N_{1}$ is produced, via freeze-in, by the decays of $\delta^{+}$while it is in equilibrium in the early Universe. In addition, we demonstrate that, thanks to the couplings between the heavier sterile neutrinos $N_{2,3}$ and $\delta^{+}$, baryogenesis via leptogenesis can be realized close to the electroweak scale. The lepton asymmetry is generated either by $N_{2,3}$-decays for masses $M_{2,3} \gtrsim$ $\mathrm{TeV}$, or by $N_{2,3}$-oscillations for $M_{2,3} \sim \mathrm{GeV}$. Experimental signatures of this scenario include an X-ray line from dark matter decays, and the direct production of $\delta^{+}$at the LHC. This model thus describes a minimal, testable scenario for neutrino masses, the baryon asymmetry, and dark matter.
\end{abstract}

\section{Introduction}

Sterile neutrinos, that is, fermions singlet under the $S U(3) \times$ $S U(2) \times U(1)$ gauge symmetry, are a very well-motivated extension of the standard model (SM). On the theoretical side, they are a prediction of left-right symmetric theories, they allow to gauge $B-L$ by removing its anomaly and they are necessary in $S O(10)$ grand unification. On the phenomenological side, they provide a non-vanishing mass to the active SM neutrinos, they allow to realize baryogenesis via leptogenesis, and they are also a viable candidate for dark matter, as long as their mass lies in the $\mathrm{keV}$ range. In that case, they are naturally long-lived so that, unlike dark matter candidates at the electroweak scale, no additional symmetries are required to stabilize them. Thus, sterile neutrinos

\footnotetext{
a e-mail: carlos.yaguna@uni-muenster.de
}

can provide a simple solution to the three open problems of the SM: neutrino masses, the baryon asymmetry, and the dark matter.

The minimal model addressing these three issues requires one sterile neutrino $N_{1}$ at the keV scale as dark matter candidate [1], and two additional sterile neutrinos $N_{2,3}$ for leptogenesis, which is induced either by $N$-decays, for sterile neutrino masses above the $\mathrm{TeV}$ scale [2], or by $N$-oscillations, for sterile neutrino masses at the $\mathrm{GeV}$ scale [3,4]. Light active neutrino masses are easily generated, via the seesaw mechanism, provided the sterile neutrino masses are significantly larger than about $1 \mathrm{eV}$. Thus, one may argue that no new physics is needed above the electroweak scale to explain neutrino masses, baryogenesis, and dark matter, defining the so-called " $v$ minimal standard model" ( $v$ MSM) [5]. Such a model is undoubtedly economical and very predictive, by reason of the small number of degrees of freedom it contains, but it is also strongly constrained. Present experiments, in fact, already exclude the minimal scenario for sterile neutrino dark matter within the $v$ MSM [6]. In the region of mass and mixing angle compatible with current observations, in particular the X-ray bounds [7-9] and the $\mathrm{Ly}_{\alpha}$ forest data [10-12], one cannot produce a large enough population of sterile neutrinos from standard active-sterile oscillations [1]. The only way out within the $\nu$ MSM is to generate the dark matter neutrinos via resonant active-sterile oscillations triggered by large initial lepton asymmetries $[13,14]$, but that mechanism requires a high degree of fine-tuning $[6,15]$. In addition, leptogenesis via $N_{2,3}$-oscillations within the $\nu$ MSM also requires a significant tuning of parameters, in particular a strong mass degeneracy between $N_{2}$ and $N_{3}$ [6].

It is important, therefore, to consider alternative ways of realizing leptogenesis and producing sterile neutrinos within extensions of the $v$ MSM. Indeed, most extensions of the SM that are theoretically well-motivated contain new degrees of freedom beside the sterile neutrinos, which may modify significantly the phenomenology of the vMSM. For leptogen- 
esis, the required tuning of parameters can be released by introducing a second Higgs doublet, as recently studied in [16]. Regarding dark matter, several other possibilities have been considered for the production of the sterile neutrinos. They include the production through inflaton decay [17-19], thermal overproduction followed by entropy dilution [20], and the production via the decays of either a neutral scalar in thermal equilibrium [21], or a frozen-in neutral scalar [22].

In this paper we advocate an extension of the SM by three sterile neutrinos, $N_{i}$, plus a charged scalar, $\delta^{+}$, both of which are naturally present in left-right symmetric or unified extensions of the SM [23]. The charged scalar interacts with SM leptons, both doublets and singlets, and with sterile neutrinos, and its mass can be as low as allowed by current collider bounds-about $200 \mathrm{GeV}$. We will show that the interplay between $N_{i}$ and $\delta^{+}$modifies the dark matter phenomenology, enables new realizations of baryogenesis, and gives rise to novel experimental signatures at colliders. The decays of $\delta^{+}$, in fact, constitute a new mechanism for the production of sterile neutrino dark matter in the early Universe. An advantage of this mechanism is that the resulting relic density does not depend on the active-sterile mixing angle, allowing to satisfy the strong bounds derived from X-ray observations [79]. Besides, the charged scalar induces independent sources of leptogenesis that are effective at scales close to the electroweak scale. If the charged scalar mass were accessible at colliders, the present scenario for dark matter and baryogenesis would be testable in a direct way.

The rest of the paper is organized as follows. In Sect. 2 we introduce the model and fix our notation. Section 3 deals with dark matter production via the decays of $\delta^{+}$. We obtain, in particular, the dark matter relic density as a function of the parameters of the model. Baryogenesis via leptogenesis is discussed in Sect.4. We examine two different ways to generate a lepton asymmetry, and outline the region of the parameter space that successfully realizes baryogenesis in each case. The most interesting experimental signatures of this model are discussed in Sect. 5. Section 6 briefly elaborates on the flavour structure of the masses and Yukawa couplings of the model. We present our conclusions in Sect. 7.

\section{The model}

When gauge singlet chiral fermions $N_{R i}$ are added to the SM, they have in general a Majorana mass term and a Yukawa coupling to the SM lepton doublets,

$$
\begin{aligned}
\mathscr{L}_{N}= & \overline{N_{R i}} i \gamma^{\mu} \partial_{\mu} N_{R i} \\
& +\left[-\frac{1}{2} \overline{\left(N_{R i}\right)^{c}}\left(M_{N}\right)_{i j} N_{R j}-\overline{l_{L \alpha}}\left(y_{\nu}\right)_{\alpha i} N_{R i} \tilde{H}+\text { h.c. }\right] .
\end{aligned}
$$

When a scalar field $\delta^{+}$, singlet under $S U(2)$ and with electromagnetic charge one, is added to the SM, it has in general, besides its gauge and self-interactions, a quartic coupling to the Higgs doublet and an antisymmetric Yukawa coupling to two lepton doublets,

$$
\begin{aligned}
\mathscr{L}_{\delta}= & D_{\mu} \delta^{+} D^{\mu} \delta^{-}-M_{\delta}^{2} \delta^{+} \delta^{-}-\frac{1}{2} \lambda_{\delta}\left(\delta^{+} \delta^{-}\right)^{2} \\
& -\lambda_{\delta H} \delta^{+} \delta^{-} H^{\dagger} H+\left[-\overline{l_{L \alpha}}\left(y_{L}\right)_{\alpha \beta}\left(i \sigma_{2}\right)\left(l_{L \beta}\right)^{c} \delta^{-}+h . c .\right]
\end{aligned}
$$

When both the sterile neutrinos $N_{R i}$ and the charged scalar $\delta^{+}$ are present, there is an additional Yukawa coupling involving the SM lepton singlets,

$\mathscr{L}_{\delta N}=-\overline{\left(e_{R \alpha}\right)^{c}}\left(y_{R}\right)_{\alpha i} N_{R i} \delta^{+}+$h.c.

These three terms, Eqs.(1)-(3), plus the well-known SM ones, constitute the Lagrangian considered in this paper. It is the most general Lagrangian consistent with the $S U(3) \times$ $S U(2) \times U(1)$ gauge symmetry and with the addition of the singlet fermions and the charged scalar. Without loss of generality, we adopt the basis where $M_{N}$ and the charged lepton mass matrix are real and diagonal. For our following discussion, the parameters of greater relevance are the masses of the singlet fermions $\left(M_{i}, i=1,2,3\right)$ and of the charged scalar $\left(M_{\delta}\right)$, and the $3 \times 3$ Yukawa matrices $y_{v}$ and $y_{R}$.

In this model active neutrino masses are not affected by the existence of $\delta^{+}$and are obtained via the usual seesaw mechanism: $m_{v} \simeq y_{v} M_{N}^{-1} y_{v}^{T} v^{2}$, with $v \simeq 174 \mathrm{GeV}$. In Sect. 3 we will identify the sterile neutrino $N_{1}$ with a multi-keV dark matter candidate, with very small mixing angles with active neutrinos, corresponding to tiny neutrino Yukawa couplings, $\left(y_{v}\right)_{\alpha 1} \lesssim 3 \times 10^{-13}$. These tiny couplings give rise to a lightest neutrino mass $m_{v}^{\text {lightest }} \lesssim 10^{-6} \mathrm{eV}$. The much larger solar and atmospheric neutrino mass scales are generated instead by the seesaw contribution of the two heavier sterile neutrinos, $N_{2,3}$. The active neutrino mass spectrum is thus hierarchical (either normal or inverted), with one neutrino remaining essentially massless.

The generation of the dark matter energy density and of the baryon asymmetry have been extensively studied in the context of the SM extended with sterile neutrinos only [6]. The SM extension with sterile neutrinos and $\delta^{+}$was considered in [23]. It was shown that, taking the sterile neutrinos $N_{i}$ as light as a few TeVs and heavier than $\delta^{+}$, one can generate the observed baryon asymmetry via leptogenesis, with no need to enhance resonantly the $\mathrm{CP}$ asymmetry.

We explore, instead, the possibility that the lightest singlet fermion, $N_{1}$, has a multi-keV mass, $M_{1} \ll M_{\delta}$, and accounts for the observed dark matter density. In this framework, the existence of $\delta^{+}$offers an alternative way of producing dark matter that has not been studied before in the 
literature. Moreover, we will show that this different mass spectrum is still compatible with the leptogenesis mechanism proposed in [23] and, in addition, we will identify a different mechanism for leptogenesis in the presence of $\delta^{+}$ based on $N_{2,3}$-oscillations.

\section{Dark matter production from $\delta^{+}$decays}

In this model, the only particle that can play the role of dark matter is the lightest singlet fermion, $N_{1}$. It is important, therefore, to determine how it is produced in the early Universe and whether it can account for the observed dark matter density while respecting all other experimental constraints. In this section, after briefly reviewing the standard scenario for sterile neutrino dark matter, we propose a new mechanism for dark matter production via the decays of $\delta^{+}$.

In the absence of $\delta^{+}$, our model is described by the seesaw Lagrangian, Eq. (1), and the region of the parameter space compatible with dark matter is the one of the $\nu$ MSM. It features a singlet fermion $\left(N_{1}\right)$ with a mass at the $\mathrm{keV}$ scale, the dark matter particle, and two heavier singlets, $N_{2,3}$. In the $\nu$ MSM, dark matter is produced at temperatures of order 100 $\mathrm{MeV}$ via active-sterile neutrino mixing. The required effective mixing angle, $\theta_{1}$, is necessarily small and is related to the Yukawa couplings by $\theta_{1}^{2}=\sum_{\alpha=e, \mu, \tau}\left|\left(y_{v}\right)_{\alpha 1}\right|^{2} v^{2} / M_{1}^{2}$. Detailed numerical studies have shown that the observed dark matter density can be reproduced in two different cases [6]. In the non-resonant case (also known as the DodelsonWidrow mechanism [1]), dark matter neutrinos are thermally and non-resonantly produced with a smooth distribution of momenta. This mechanism yields the minimal amount of dark matter that can be obtained for a given mass and mixing angle. For dark matter masses between $1 \mathrm{keV}$ and 10 $\mathrm{keV}$, the mixing angle required to explain the dark matter density is $\sin ^{2}\left(2 \theta_{1}\right) \sim 10^{-8}-10^{-9}$, as shown e.g. in figure 2 of [6]. These parameters are not consistent with current data [24], which imply $M_{1} \lesssim 3-4 \mathrm{keV}$ from the X-ray line bound [7-9] and $M_{1} \gtrsim 8 \mathrm{keV}$ from the $\mathrm{Ly}_{\alpha}$ forest observations [10-12]. The other case in which the dark matter density can be explained, and the only one that is currently viable in the $\nu \mathrm{MSM}$, is resonant production (also known as the Shi-Fuller mechanism [13]). In it, the dark matter production rate is resonantly amplified by the presence of a lepton chemical potential in the plasma, which enhances the production for particular momenta as they pass through the resonance, giving rise to a non-thermal momentum distribution that is colder than that obtained in the non-resonant case. To explain the observed dark matter density, a lepton asymmetry $\left|\mu_{\alpha}\right| \gtrsim 8 \times 10^{-6}$ at $T \sim 100 \mathrm{MeV}$ is required [14], where $\mu_{\alpha}=n_{\alpha} / s, s$ is the entropy density of the Universe, and $n_{\alpha}$ is the total number density of active leptons of flavour $\alpha$. In principle, this large lepton asymmetry can be generated within the $v \mathrm{MSM}$ via CP-violating oscillations of $N_{2}$ and $N_{3}$, but only if their mass difference, $M_{3}-M_{2}$, is fine-tuned to the order of $10^{-11}[15,25]$.

The existence of $\delta^{+}$allows a new production mechanism for sterile neutrinos in the early Universe. Indeed, thanks to the interaction term in Eq. (3), the decays of the charged scalars can produce sterile neutrinos, $\delta^{+} \rightarrow N_{1} \ell^{+}$. This decay is a thermal process that takes place while $\delta^{+}$is in equilibrium and it is an example of the so-called freeze-in scenario [26] for dark matter production. The defining feature of freeze-in is that the dark matter interactions are so weak that they never reach thermal equilibrium in the early Universe. As a result, the dark matter abundance is negligible at high temperatures and slowly increases as the Universe cools down. The production ceases when the freeze-in temperature is reached, and the dark matter abundance remains constant from then on.

The $N_{1}$ yield, $Y_{N_{1}}(T)=n_{N_{1}}(T) / s(T)$, from $\delta^{+}$decays is obtained by solving the following Boltzmann equation [26]

$s T \frac{d Y_{N_{1}}}{d T}=-\frac{\gamma_{N_{1}}(T)}{H(T)}$

where $s$ is the entropy density, $H$ is the expansion rate and $\gamma_{N_{1}}(T)$ is the thermally-averaged production rate. We have that

$$
\begin{aligned}
\gamma_{N_{1}}(T)= & \frac{M_{\delta}^{2} T}{2 \pi^{2}} K_{1}\left(M_{\delta} / T\right) \\
& \times \sum_{\alpha}\left[\Gamma\left(\delta^{-} \rightarrow N_{1} \ell_{\alpha}^{-}\right)+\Gamma\left(\delta^{+} \rightarrow N_{1} \ell_{\alpha}^{+}\right)\right]
\end{aligned}
$$

where $K_{1}(x)$ is the Bessel function of the second kind and the sum runs over the different lepton flavours. Since the lepton and $N_{1}$ masses are negligible compared to $M_{\delta}$, the $\delta^{+}$decay rates are calculated as

$\Gamma\left(\delta^{-} \rightarrow N_{1} \ell_{\alpha}^{-}\right)=\Gamma\left(\delta^{+} \rightarrow N_{1} \ell_{\alpha}^{+}\right)=\frac{M_{\delta}\left|\left(y_{R}\right)_{\alpha 1}\right|^{2}}{16 \pi}$

and their sum over flavours can be conveniently written as

$$
\begin{aligned}
& \sum_{\alpha}\left[\Gamma\left(\delta^{-} \rightarrow N_{1} \ell_{\alpha}^{-}\right)+\Gamma\left(\delta^{+} \rightarrow N_{1} \ell_{\alpha}^{+}\right)\right]=\frac{M_{\delta}}{8 \pi}\left(y_{R}^{\dagger} y_{R}\right)_{11} \\
& \quad=\frac{M_{\delta}}{8 \pi} y_{R 1}^{2},
\end{aligned}
$$

where we have defined $y_{R 1}$ as the combination of couplings that determines the production of $N_{1}$ via $\delta^{+}$decays. Using $s(T)=2 \pi^{2} g_{s} T^{3} / 45, H(T)=1.66 \sqrt{g_{\rho}} T^{2} / M_{P l}$, and $x \equiv$ $M_{\delta} / T$ we can solve Eq. (4) as

$Y_{N_{1}}\left(T_{0}\right)=\frac{45}{(1.66) 32 \pi^{5} g_{s} \sqrt{g_{\rho}}} \frac{M_{P l} y_{R 1}^{2}}{M_{\delta}} \int_{x_{\min }}^{x_{\max }} x^{3} K_{1}(x) d x$, 
where $T_{0}$ is the temperature today and we used $Y(T \gg$ $\left.M_{\delta}\right)=0$ as our initial condition. Integrating this equation from $x_{\max }=\infty$ to $x_{\min }=0$ and setting $g_{s} \approx g_{\rho} \approx 100$ yields

$Y_{N_{1}}\left(T_{0}\right) \approx 1.6 \times 10^{-5}\left(\frac{y_{R 1}}{10^{-8}}\right)^{2}\left(\frac{1 \mathrm{TeV}}{M_{\delta}}\right)$.

The $N_{1}$ relic density is then

$\Omega_{N_{1}} h^{2} \approx 0.11\left(\frac{M_{1}}{\mathrm{keV}}\right)\left(\frac{y_{R 1}}{5 \times 10^{-8}}\right)^{2}\left(\frac{1 \mathrm{TeV}}{M_{\delta}}\right)$

Thus, a keV sterile neutrino can explain the observed dark matter density via $\delta^{+}$decays if $M_{\delta} \sim 1 \mathrm{TeV}$ and $y_{R 1} \sim$ few $\times 10^{-8}$. This analytical result is in very good agreement with the numerical calculations we did for similar models of freeze-in dark matter $[27,28]$.

A crucial feature of Eq. (10) is that, unlike the production in the $v \mathrm{MSM}$, it does not depend on the active-sterile mixing angle $\theta_{1}$ that determines the decay rate of the sterile neutrino. Hence, one can take $\theta_{1}$ small enough to be in agreement with the X-ray bounds without affecting the predicted dark matter density. In other words, in this model one can decouple the dark matter production (determined by the $y_{R}$ couplings) from the dark matter decay (determined by $y_{v}$ via mixing angles), opening new viable regions and alleviating the experimental constraints on sterile neutrino dark matter. In particular, $M_{1}$ can be larger than the $\mathrm{keV}$ scale, as long as the dark matter sterile neutrino is sufficiently long-lived. Since $\Gamma_{N_{1}} \propto M_{1}^{5} \sin ^{2} \theta_{1}$, one cannot exclude, for example, the possibility of vanishing active-sterile mixing, $\theta_{1}=0$, which would allow masses as high as $1 \mathrm{MeV}$. Above that value, the decay $N_{1} \rightarrow v e^{+} e^{-}$becomes kinematically available and can proceed through the couplings $y_{R}$ and $y_{L}$, even for $\theta_{1}=0$. In that case, the strong constraints coming from indirect searches of electrons and positrons come into play, but they compete with the smallness of $y_{R 1}$, determined by Eq. (10), and of the Yukawa matrix $y_{L}$, that can be very small too.

Regarding structure formation, the sterile neutrinos produced via $\delta^{+}$decays are colder than those obtained in the $\nu$ MSM [29,30] because the decays take place at a temperature $T \sim M_{\delta}$ much higher than the QCD scale $(\sim 150 \mathrm{MeV})$. In fact, their free-streaming length and phase space density are identical to those of the so-called chilled sterile neutrinos studied in [31]. Consequently, the Lyman- $\alpha$ bounds on the sterile neutrino mass in our scenario are significantly weaker, allowing for a dark matter mass $M_{1}$ smaller than in the $v$ MSM [32].

We assume in the following that the decays of the charged scalars are the dominant source of sterile neutrinos, so that Eq. (10) is satisfied. This will be the case in the absence of a large lepton asymmetry and for small mixing angles, $\sin ^{2} 2 \theta_{1}<10^{-9}$. Next, we show that this assumption is consistent with baryogenesis, and that it leads to new experimental signatures.

\section{Baryogenesis via leptogenesis with $\delta^{+}$}

In this section we will discuss leptogenesis in the presence of the charged scalar field $\delta^{+}$. We will briefly review the two basic mechanism for leptogenesis in the minimal scenario with sterile neutrinos only: $N$-decays and $N$-oscillations. We will show that both mechanisms are still operative when one replaces the Yukawa coupling $y_{v}$ in Eq. (1) with $y_{R}$ in Eq. (3), that is, when the role of the SM Higgs doublet $H$ is played by $\delta^{+}$, and the role of the SM lepton doublet $l_{L}$ is played by the SM lepton singlet $e_{R}$.

The baryon asymmetry of the Universe can be generated from a lepton asymmetry, as long as the latter is present before the electroweak phase transition, which occurs at a temperature $T_{E W} \simeq 150 \mathrm{GeV}$. Above this temperature the $(B+L)$ violating electroweak sphalerons are in thermal equilibrium, thus converting efficiently leptons into baryons. When the $\mathrm{SM}$ is extended by sterile neutrinos, one can generate a lepton asymmetry, provided that the set of couplings $\left(M_{N}\right)_{i j}$, $\left(y_{\nu}\right)_{\alpha i}$ and $\left(y_{R}\right)_{\alpha i}$ violates the $C P$ symmetry, and (some of) these couplings are out-of-equilibrium at some epoch before $T_{E W}$.

\subsection{Leptogenesis from $N$-decays}

The traditional leptogenesis mechanism [2] assumes a Majorana mass matrix $M_{N}$ for two (or more) sterile neutrinos, with eigenvalues $M_{i}$ larger than the electroweak scale, and it relies on the out-of-equilibrium decays $N_{i} \rightarrow H l_{L \alpha}$, at temperatures just below $M_{i}$. As $N_{1}$ plays the role of keV-scale dark matter candidate, the relevant decays are those of $N_{2}$, with $T_{E W}<M_{2}<M_{3}$. Since $M_{2}$ violates lepton number, the $C P$-asymmetry that is generated in the decays is also a lepton asymmetry, or equivalently a $(B-L)$-asymmetry. After all the $N_{2}$ particles decayed, the produced $(B-L)$ asymmetry remains constant. In this scenario, the couplings that control leptogenesis are the same Yukawa couplings, $y_{\nu}$, that determine the active neutrino masses. As a consequence, a sufficient amount of baryon asymmetry can be generated only for $M_{2} \gtrsim 10^{8} \mathrm{GeV}$ approximately. ${ }^{1}$ Thus, no direct test of this scenario can be envisaged.

\footnotetext{
${ }^{1}$ This lower bound on the leptogenesis scale was pointed out in [33] and its exact value depends on various parameters, in particular the initial $N_{1}$ abundance [34]. It can be evaded if one allows for resonance effects, that require a strong degeneracy among the sterile neutrino mass eigenstates [35].
} 
In the presence of a charged scalar $\delta^{+}$, an analog source of leptogenesis is provided by the decays $N_{i} \rightarrow \delta^{+} e_{R \alpha}$, mediated by the Yukawa coupling $y_{R}$, as shown in [23]. Here we adapt the results of that paper to our new context, where the dark matter particle is the keV-scale sterile neutrino $N_{1}$. We can safely neglect the presence of $N_{1}$ for the computation of the lepton asymmetry because its interaction rates (in particular those violating lepton number) are strongly outof-equilibrium above $T_{E W}$, due to the extreme smallness of $M_{1},\left(y_{R}\right)_{\alpha 1}$ and $\left(y_{\nu}\right)_{\alpha 1}$. Thus, at least two extra sterile neutrinos $N_{2,3}$ heavier than $\delta^{+}$are needed to generate the $C P$ asymmetry. Taking for simplicity $M_{3} \gg M_{2} \gg M_{\delta}$, the $\mathrm{CP}$-asymmetry in $\mathrm{N}_{2}$-decays is given by

$\epsilon_{N_{2}}=\frac{1}{8 \pi} \frac{\operatorname{Im}\left[\sum_{\alpha}\left(y_{R}\right)_{\alpha 2}\left(y_{R}\right)_{\alpha 3}^{*}\right]^{2}}{\sum_{\alpha}\left(y_{R}\right)_{\alpha 2}\left(y_{R}\right)_{\alpha 2}^{*}} \frac{M_{2}}{M_{3}}$,

where we assumed that the charged scalar lepton number is $L\left(\delta^{+}\right)=-2$, since the dominant decay mode is $\delta^{+} \rightarrow e^{+} \bar{\nu}$, through the Yukawa coupling $y_{L}$ in Eq. (2). ${ }^{2}$ To reproduce the observed baryon asymmetry two basic conditions are required [23]: a minimal value for $\epsilon_{N_{2}}$ assuming no washout,

$\frac{\left|\operatorname{Im}\left[\sum_{\alpha}\left(y_{R}\right)_{\alpha 2}\left(y_{R}\right)_{\alpha 3}^{*}\right]^{2}\right|}{\sum_{\alpha}\left(y_{R}\right)_{\alpha 2}\left(y_{R}\right)_{\alpha 2}^{*}} \gtrsim 2 \cdot 10^{-6} \frac{M_{3}}{M_{2}}$,

and $N_{2}$-decays out-of-equilibrium at $T=M_{2}$ to avoid large washout from inverse decays,

$\sum_{\alpha}\left(y_{R}\right)_{\alpha 2}\left(y_{R}\right)_{\alpha 2}^{*} \lesssim 10^{-13} \frac{M_{2}}{1 \mathrm{TeV}}$.

Thus, one can realize leptogenesis for $M_{2}$ as small as a few TeVs, as long as $N_{2,3}$ have hierarchical Yukawa couplings: $\left|\left(y_{R}\right)_{\alpha 2}\right| \lesssim 3 \times 10^{-7}$ and $\left|\left(y_{R}\right)_{\alpha 3}\right| \gtrsim 10^{-3} \sqrt{M_{3} / M_{2}}$. It is worth reminding that, when $M_{2}$ approaches the electroweak scale, a sufficiently large CP-asymmetry implies in general strong $(B-L)$-washout rates, in particular those mediated by $N_{3}$ off-shell, unless special conditions are realized. A neat, model-independent discussion of the lower bound on the leptogenesis scale can be found in $[36,37]$. In the present model with $M_{2} \sim$ a few TeVs, the washout can be Boltzmann suppressed by raising $M_{\delta}$ sufficiently close to $M_{2}$, and taking a sufficiently large coupling $y_{L}$ in Eq. (2) to avoid washout from the asymmetry stored in $\delta^{+} .3$

Note that the asymmetries generated by $N_{2}$-decays through the Yukawa coupling matrix $y_{v}$ become negligible in the

\footnotetext{
2 The asymmetry changes by an order one factor if $y_{L}$ is negligibly small and $\delta^{+}$undergoes slower three-body decays [23].

3 To reduce the washout, one can also delay $N_{2}$-decays by taking $\left(y_{R}\right)_{\alpha 2}$ very small, but in this case one may need to produce the initial thermal density of $N_{2}$ by some other interaction.
}

region $M_{2} \ll 10^{8} \mathrm{GeV}$ due to the seesaw relation. The $y_{R^{-}}$ entries, on the other hand, are not constrained by the light neutrino masses, and leptogenesis can work close to the electroweak scale as described above. This scenario is, therefore, easier to test directly, by the observation of $\delta^{+}$(and possibly $N_{2}$ ) at colliders, as discussed in Sect. 5.

\subsection{Leptogenesis from $N$-oscillations}

A complementary mechanism for leptogenesis [3] relies on $N$-oscillations rather than on $N$-decays. It also requires two (or more) sterile neutrinos, coupled to the SM through the Yukawa matrix $y_{v}$. In the early Universe, the thermal population of lepton doublets $l_{L \alpha}$ produces, through small, out-ofequilibrium couplings $\left(y_{v}\right)_{\alpha i}$, a coherent superposition $N_{\alpha}$ of the sterile neutrino mass eigenstates $N_{i}$. These sterile neutrinos, at temperatures much larger than their masses, coherently oscillate among the different flavours $\alpha$. Such oscillations conserve lepton number (the $N_{\alpha}$ conserve their helicity), but violate lepton flavour numbers. If the $C P$-symmetry is also violated, one generates non-zero flavour asymmetries between the opposite helicities of the $N_{\alpha}$. The asymmetry in the flavour $\alpha$ is transferred efficiently to $l_{L \alpha}$, as long as the coupling $\left(y_{v}\right)_{\alpha i}$ goes into equilibrium for some $i$. Since the total lepton asymmetry, that is, the sum over $\alpha$ of the flavour asymmetries vanishes, one needs that some but not all flavours go into equilibrium before $T_{E W}$, so that a net lepton asymmetry remains stored in the sterile neutrino sector, and an opposite one is available in the SM sector to be transferred to the baryons by electroweak sphalerons. The Yukawa (out-of-)equilibrium condition at $T_{E W}$ reads

$$
\begin{aligned}
\left|\left(y_{\nu}\right)_{\alpha i}\right| & \gtrsim 10^{-7} \quad \text { for some } \alpha \text { and } i, \quad\left|\left(y_{\nu}\right)_{\beta i}\right| \\
& \lesssim 10^{-7} \quad \text { for some } \beta \neq \alpha, \forall i .
\end{aligned}
$$

A number of additional constraints, on the size of $y_{v}$-entries and on the values of $M_{i}$, must be satisfied for this leptogenesis mechanism to work. To facilitate the comparison between the scenarios with and without the charged scalar, we will describe them in some detail. Due to the dark matter constraint, $N_{1}$ plays no role in leptogenesis and the asymmetry must be generated by $N_{2}$ and $N_{3}$ only. ${ }^{4}$

Successful leptogenesis implies a few upper bounds on the $y_{v}$-entries, beside the flavour-dependent one in Eq. (14). First of all, note that the Majorana masses $M_{i}$ violate lepton number. Therefore, they play the role of washout in this scenario, as they transform opposite helicities of the $N_{\alpha}$ into one another. The condition to keep the lepton number violation rate out-of-equilibrium down to $T_{E W}$ is approximately

\footnotetext{
${ }^{4}$ Note that the seesaw lagrangian can violate $C P$ with only two sterile neutrinos, as it contains three physical phases, one combination of them being relevant in oscillations.
} 
$\left|\left(y_{\nu}\right)_{\alpha i}\right| \lesssim 10^{-5} \frac{\mathrm{GeV}}{M_{i}}, \quad \forall \alpha$ and $\forall i$

which combined with Eq. (14) implies sterile neutrino masses below the electroweak scale, $M_{i} \lesssim 100 \mathrm{GeV}^{5}{ }^{5}$ In addition, for the case of two sterile neutrinos $N_{2,3}$, analytic and numerical studies $[4,16,39]$ show that a sufficient baryon asymmetry requires a strong degeneracy between $M_{2}$ and $M_{3}$, with $\Delta M / M \lesssim 10^{-5}$. This comes from the interplay of a number of subtle effects: the oscillation time increases as $\Delta M$ decreases, and this allows for larger asymmetries because the Yukawa interaction rates are closer to equilibrium at later times; note also that larger Yukawa couplings (for some but not all the flavours) enhance the flavour asymmetries, but also tend to spoil the coherence of the sterile neutrinos, as active-sterile transitions may become faster than the oscillation time. We extrapolate the resulting constraint from figure 7 of [6], that can be written as

$\left|\left(y_{\nu}\right)_{\alpha i}\right| \lesssim 2 \times 10^{-6}\left(\frac{M_{i}}{\mathrm{GeV}}\right)^{1 / 2} \quad \forall \alpha$ and $\forall i$

The masses $M_{2,3}$ are below collider energies, but it is difficult to produce them directly since they have small couplings. Still, the mixing with active neutrinos can be sufficiently large to have an observable effect in various neutrino experiments [40]. The resulting upper bound on active-sterile mixing is also shown in figure 7 of [6]. An experimental proposal to improve significantly the present bound can be found in [41]. In less minimal models, e.g. with three GeV scale sterile neutrinos, there are good detection perspectives in meson decay experiments [42].

On the other hand, several observables other than the baryon asymmetry put lower bounds on the $y_{v}$-entries, that add to the one in Eq. (14). The lower bound on active neutrino masses, $\left|\left(m_{v}\right)_{\alpha \beta}\right| \gtrsim 0.025 \mathrm{eV}$ for some $\alpha$ and $\beta$, implies a lower bound on the neutrino Yukawa couplings through the seesaw formula,

$\left|\left(y_{\nu}\right)_{\alpha i}\right| \gtrsim 2 \times 10^{-8}\left(\frac{M_{i}}{\mathrm{GeV}}\right)^{1 / 2}$ for some $\alpha$ and $i$.

Indeed, since $N_{1}$ gives a negligible contribution to $m_{v}$, one active neutrino is approximately massless, therefore $\left|\left(m_{\nu}\right)_{\alpha \beta}\right| \lesssim 0.05 \mathrm{eV}$ for all $\alpha$ and $\beta$. Then, the right-hand

\footnotetext{
5 We remark that most numerical studies in the literature assume that the lepton number violating rates mediated by $M_{i}$ are negligible; in view of the size of the Yukawa couplings relevant for leptogenesis, this assumption seems to be justified only for $M_{i} \lesssim \mathrm{GeV}$. For larger $M_{i}$, one should include the lepton number violating rates in the Boltzmann equations. In this regime, it remains conceivable that the $C P$-asymmetries are large enough to compensate the relatively strong washout. This possibility was recently entertained to make this scenario work even for $M_{i}$ larger than $T_{E W}[38]$.
}

side of Eq. (17) provides the natural value of the Yukawa couplings for $i=2,3$ : the largest $\left(y_{\nu}\right)_{\alpha i}$ are, the strongest is the cancellation needed among the contributions of $N_{2}$ and $N_{3}$ to $m_{v}$. These GeV-scale sterile neutrinos should decay (e.g. in $3 v$ 's or $v e^{+} e^{-}$) before $T \simeq 1 \mathrm{MeV}$, not to spoil nucleosynthesis (see [43] for a detailed analysis). We roughly estimate this constraint as

$\left|\left(y_{\nu}\right)_{\alpha i}\right| \gtrsim 3 \cdot 10^{-8}\left(\frac{\mathrm{GeV}}{M_{i}}\right)^{3 / 2}$, for some $\alpha, \quad \forall i$

Comparing with Eq. (16), this implies $M_{i} \gtrsim 0.1 \mathrm{GeV}$. All the constraints above confirm that $N_{1}$ plays no role in leptogenesis.

Let us show that the same mechanism of leptogenesis through $N$-oscillations is operative in the presence of the charge scalar $\delta^{+}$, by replacing the role of $y_{v}$ with $y_{R}$. Analogously to the previous case, the couplings $\left(y_{R}\right)_{\alpha i}$ must be small to remain out-of-equilibrium while they slowly produce coherent sterile neutrino states. The lepton flavour asymmetries generated by $N$-oscillations are (partially) transferred to the SM lepton singlets $e_{R \alpha}$. The latter are in equilibrium with $l_{L \alpha}$ through the charged lepton Yukawa couplings (at least for $\alpha=\mu, \tau$ ), thus electroweak sphalerons transfer the asymmetries to baryons as usual.

One needs that the $y_{R}$-entries satisfy the same inequalities as the $y_{\nu}$-entries in Eqs. (14) and (15), so that some flavour asymmetries are transferred to baryons, while the others remain stored in the sterile sector, and the washout from lepton number violating scattering is small. The only differences amount to (i) order one factors to account for the singlet (doublet) nature of $e_{R}\left(l_{L}\right)$ in scattering rates; (ii) the range of temperatures where $M_{\delta}\left(M_{H}\right)$ can be neglected: note that the asymmetry is generated at some scale well above $T_{E W}$ through scattering processes mediated by $\delta^{+}(H)$.

We also expect that, when both $y_{v}$ and $y_{R}$ take values relevant for leptogenesis, there is more freedom to generate large asymmetries, e.g. because of the presence of extra $C P$-violating phases, and the requirement of a strong degeneracy between $M_{2}$ and $M_{3}$ could be relaxed. As in the case of $y_{v}$, one cannot raise too much the value of $y_{R}$-entries, as the coherence of sterile neutrino oscillations requires the scattering rate with the SM leptons to be out-of-equilibrium at the time of oscillations; this should translate in a bound similar to the one in Eq. (16). A numerical study is needed to establish more precisely the allowed parameter space, and the differences with respect to the $v$ MSM one. The effect of the two sets of Yukawa couplings could be dramatic, as is the effect of a third GeV scale sterile neutrino [42,44], or of a second Higgs doublet [16].

There is no constraint on $y_{R}$ coming from the seesaw relation, so one can take couplings smaller than in Eq. (17), slightly enlarging the region of parameters of leptogenesis. 
Perhaps more importantly, one can take $\left(y_{R}\right)_{\alpha i}$ significantly larger than the right-hand side of Eq. (17), with no need of fine-tuning to keep $m_{v}$ small. Note that the coupling $y_{R}$ does not induce any mixing with active neutrinos. Indeed, direct searches of active-sterile mixing are presently sensitive to $y_{v}$-entries much larger than in Eq. (17): if the seesaw parameters take their natural values, no direct signal of active-sterile mixing is expected.

Finally, the three-body decay rate of $N_{i}$ through the coupling $y_{R}$ is proportional to $\left|\left(y_{R}\right)_{\alpha i}\left(y_{L}\right)_{\beta \gamma}\right|^{2} / M_{\delta}^{4}$, to be compared with $\left|\left(y_{v}\right)_{\alpha i}\right|^{2} /\left(M_{i}^{2} v^{2}\right)$ for a decay through the mixing with active neutrinos. Since the latter is typically much faster, the nucleosynthesis bound applies to $y_{v}$ only, in the form of Eq. (18). Then, leptogenesis through $y_{R}$ could work even for $M_{i}<0.1 \mathrm{GeV}$, but in this region nucleosynthesis demands large values for the $y_{v}$-entries, that require strong cancellations in the seesaw.

\section{Experimental signatures for $N_{1}$ and $\delta^{+}$}

Two important differences between our model and the $v$ MSM are the additional region of the parameter space where the dark matter constraint can be satisfied and the presence of the extra charged scalar $\delta^{+}$. They both give rise to new experimental signatures that may allow to distinguish one model from the other.

\subsection{Dark matter indirect detection}

A multi-keV dark matter neutrino is inherently unstable and decays into three light neutrinos at tree-level $\left(N_{1} \rightarrow 3 v_{\alpha}\right)$ and radiatively into a light neutrino and a photon $\left(N_{1} \rightarrow v_{\alpha} \gamma\right)$. This radiative decay produces an X-ray line at $E_{\gamma} \simeq M_{1} / 2$ that can be searched for and used to constrain the model or to help determine its parameter space [45]. The presence of the additional scalar $\delta^{+}$does not affect the decay modes of the dark matter neutrino, which are still determined by its mass and its mixing with the active neutrinos, just as in the $\nu$ MSM, but it modifies the regions that are consistent with the dark matter constraint, allowing, in particular, for smaller mixing angles. This fact has important implications, as we show next.

Recently, the detection of an unidentified spectral line at about $3.5 \mathrm{keV}$ has been reported from two independent data sets $[46,47]$. Arguments in favour or against the dark matter decay interpretation of the signal can be found in [48-51]. If confirmed, that signal would provide compelling evidence for $\mathrm{keV}$-scale dark matter and, in particular, for dark matter in the form of sterile neutrinos. Within that framework, the signal can be explained if $M_{1} \simeq 7 \mathrm{keV}$ and $\sin ^{2} 2 \theta_{1} \simeq 5 \times 10^{-11}$. In the minimal scenario for sterile neutrino dark matter, where they are produced non-resonantly, such parameters lead to a relic density way below the range determined by cosmological observations. Thus, an additional source of sterile neutrinos is required. A simple possibility for that new source are the decays of the $\delta^{+}$particle, as explained in the previous section. These decays allow to decouple the dark matter production (determined by the $y_{R}$ couplings) from the dark matter decay (determined by $y_{v}$ via mixing angles), opening new viable regions consistent with all bounds. Specifically, the freeze-in production of sterile neutrinos that we have examined in this paper enables to explain the tentative $3.5 \mathrm{keV}$ line and to simultaneously account for the observed relic density, even in the absence of a large, primordial lepton asymmetry. If that line signal turns out to be spurious, $\mathrm{X}$-ray observations will continue to be the main way in which the dark matter sector of this model can be tested in the foreseeable future.

\subsection{Charged scalar searches at colliders}

The singly-charged isosinglet scalar $\delta^{+}$could be directly produced at colliders, if its mass were within their energy range. The dominant production channel is the Drell-Yan process $\psi \bar{\psi} \rightarrow \gamma / Z \rightarrow \delta^{+} \delta^{-}$, with $\psi=e$ at LEP and $\psi=q$ at Tevatron and LHC, with a partonic cross-section given e.g. in [52]. The $\delta^{+}$decays into one anti-lepton and one anti-neutrino, either through the Yukawa coupling $\left(y_{L}\right)_{\alpha \beta}$, or $\left(y_{R}\right)_{\alpha i}$ when $M_{i}<M_{\delta}{ }^{6}$

The standard experimental searches for charged scalars usually assume production and decay modes different from those above. For a singly-charged scalar $H^{+}$, the decay channel into lepton plus missing energy has been analyzed, but only for an isodoublet produced from a top-bottom vertex, as in type II two-Higgs doublet models, allowing for an effective background reduction [53,54]. Indeed, the signal over background ratio is significantly smaller in the case of $\delta^{+}$; an analysis of the cuts required to maximize the signal has been presented in section 5.2 of [55].

Presently, it appears that the best way to constrain $M_{\delta}$ is to use the experimental searches for supersymmetric particles. Since $\delta^{+}$and a right-handed slepton have the same gauge quantum numbers, when the latter is directly pair-produced and decays into a lepton and a light neutralino, it behaves very much as the former, and the same bounds apply to both particles. Recently, that setup was studied in the context of simplified supersymmetric models, for the case of a righthanded s-electron degenerate with a right-handed s-muon, decaying with branching ratio $100 \%$ into electron and muon, respectively, plus missing energy [56,57]. Assuming a similar efficiency for electron and muon detection and reducing

\footnotetext{
${ }^{6}$ In principle these couplings can all be very small, possibly leading to dominant three-body decays into $l^{+} l^{-} W^{+}$[23]. In this case $\delta^{+}$can be sufficiently long-lived to appear as a curved charged track across the whole detector.
} 
the signal by a factor two, to take into account that the $\delta^{+}$production cross-section is the same as for a single right-handed slepton, we can extract a $95 \%$ C.L. lower bound $M_{\delta} \gtrsim 200$ $\mathrm{GeV}$ at ATLAS [56] (see also the auxiliary figures 11a and 12a available online ${ }^{7}$ ) and $M_{\delta} \gtrsim 160 \mathrm{GeV}$ at CMS [57].

Note, however, that these bounds apply as long as the $\delta^{+}$ branching ratio into taus is negligible. The pair-production of s-taus decaying into taus is presently unconstrained at the LHC (see figure 9 in [58]). As a consequence, if $\delta^{+}$decays mostly into taus, its mass can be significantly smaller, say down to the LEP bound $\sim 100 \mathrm{GeV}$. Indeed, in minimal flavour models the $\delta^{+}$Yukawa couplings are hierarchical as the SM Yukawa couplings (see Sect. 6), and a large branching ratio into taus is expected.

\section{Flavour structure of the model}

We accomplished our goal of demonstrating that this model can account for dark matter, neutrino masses, and baryogenesis. That is, we have determined, in the previous sections, that there exist regions in the parameter space of the model where the masses of the new particles and their Yukawa couplings are such that these three issues are simultaneously explained. One may wonder, nonetheless, whether those values of the masses and Yukawa couplings have a generic flavour structure, or if they require a special tuning, indicating that some flavour symmetries are operative. Previous works along this line include [59-63], which focused on the generation of the $\mathrm{keV}$ scale in neutrino models, and on the flavour structure of the $v$ MSM. In this section, we do not aim to construct complete flavour models, but rather to recap the order of magnitude of the parameters needed phenomenologically, and to suggest some rationale to explain them.

In view of the hierarchical values of masses and couplings that are required for dark matter and leptogenesis, it is useful to describe the required flavour structure in terms of a $U(1)_{F}$ family symmetry, with different charges assigned to the various fermions. In this framework, the coefficient of each fermion bilinear $\psi_{L i} \psi_{L j}$ is suppressed by a power $q_{i}+q_{j}$ of a small parameter $\epsilon$ (we take the fermion $U(1)_{F^{-}}$ charges to be positive). One may assume some underlying flavour dynamics, that generates $\epsilon=\langle\phi\rangle / \Lambda_{F} \ll 1$, that is, the vev of a spurion field with $q_{\phi}=-1$, over the cutoff of the flavour theory.

We need $M_{1} \ll M_{2} \sim M_{3}$. Note that in the $v$ MSM a strong degeneracy of $M_{2}$ and $M_{3}$ is required, while in our scenario this constraint is relaxed. The straightforward way to realize this pattern is to take $q_{N 1}>q_{N 2}=q_{N 3}$, so that $M_{1} / M_{2} \sim \epsilon^{2 n}$, with $n \equiv q_{N 1}-q_{N 2}$. For $N$-oscillation

\footnotetext{
7 https://atlas.web.cern.ch/Atlas/GROUPS/PHYSICS/PAPERS/ SUSY-2013-11/.
}

( $N$-decay) leptogenesis, one needs $\epsilon^{2 n} \sim 10 \mathrm{keV} / \mathrm{GeV}$ $=10^{-5}\left(\epsilon^{2 n} \lesssim 10 \mathrm{keV} / \mathrm{TeV}=10^{-8}\right)$. This charge assignment implies automatically a hierarchy $\epsilon^{n}: 1: 1$ among the three columns of the matrix $y_{R}$ (as well as of $y_{\nu}$ ), in other words, $\left(y_{R}\right)_{\alpha i}^{2} /\left(y_{R}\right)_{\alpha j}^{2} \sim M_{i} / M_{j}$. Since this relation is not satisfied in certain regions of parameters relevant for dark matter, leptogenesis and neutrino masses, an additional flavour symmetry must be operative. The Yukawa couplings can be further suppressed by introducing parity symmetries $Z_{2}^{(i)}: N_{i} \rightarrow-N_{i}$, that allow for $M_{i}$ but forbid $\left(y_{\nu}\right)_{\alpha i}$ and $\left(y_{R}\right)_{\alpha i}$; these couplings must then be proportional to a small symmetry-breaking parameter $\epsilon_{i}$. To suppress $\left(y_{v}\right)_{\alpha i}$ and not $\left(y_{R}\right)_{\alpha i}$ (or vice versa), one may argue that under the same parity the combination $e_{R \alpha} \delta^{+}$(or $l_{L \alpha} H$ ) is also odd. ${ }^{8}$

Coming to the $U(1)_{F}$ charges of the three families of lepton doublets and singlets, $q_{L \alpha}$ and $q_{R \alpha}$, first of all they determine the hierarchy of the charged lepton masses, $m_{e}: m_{\mu}$ : $m_{\tau} \sim \epsilon^{q_{L e}+q_{R e}}: \epsilon^{q_{L \mu}+q_{R \mu}}: \epsilon^{q_{L \tau}+q_{R \tau}}$. In turn, the hierarchy among the rows of $y_{R}\left(y_{v}\right)$ is determined by the charges $q_{R \alpha}$ $\left(q_{L \alpha}\right)$. Note that the charges of $l_{L \alpha}$ and $e_{R \alpha}$ are important for charged lepton and active neutrino masses, as well as for leptogenesis from $N$-oscillations; however they are not very relevant for dark matter production nor for leptogenesis from $N$-decays.

Let us confront these simple flavour symmetries with the values of the parameters needed for dark matter, neutrino masses and leptogenesis. The freeze-in of the desired amount of $N_{1}$ from $\delta^{+}$decays requires $\sum_{\alpha}\left|\left(y_{R}\right)_{\alpha 1}\right|^{2} \simeq 2.5 \times$ $10^{-24} M_{\delta} / M_{1}$ (see Eq. (10)). The $N_{1}$-production from activesterile oscillations is negligible for $\left|\left(y_{v}\right)_{\alpha 1}\right| \lesssim 10^{-13}\left(M_{1} / \mathrm{keV}\right)$ (barring a large primordial lepton asymmetry). To generate large enough active neutrino masses, Eq. (17) must be satisfied. Clearly $N_{1}$ does not contribute significantly to $m_{v}$ because of the dark matter constraint, therefore $N_{2}$ and $N_{3}$ are responsible to generate the atmospheric and solar mass scales. Since $\sqrt{\Delta m_{\text {sol }}^{2} / \Delta m_{\text {atm }}^{2}} \simeq 0.2$, Eq. (17) must hold for both $i=2$ and 3, up to a factor of a few. Coming to leptogenesis, the two mechanisms discussed in Sect. 4 correspond to two very different regions of parameters that we discuss in turn.

In the case of leptogenesis from $N$-decays through the $y_{R}$-interaction, one requires $M_{2,3}>\mathrm{TeV}$, as well as $\left|\left(y_{R}\right)_{\alpha 3}\right|>10^{-3} \sqrt{M_{3} / M_{2}}$ for at least one $\alpha$, and $\left|\left(y_{R}\right)_{\alpha 2}\right|<$ $10^{-4} \sqrt{M_{2} /\left(10^{8} \mathrm{GeV}\right)}$ for all $\alpha$. For definiteness, let us take $M_{1} \sim \mathrm{keV}, M_{\delta} \sim \mathrm{TeV}$ and $M_{2,3} \sim 100 \mathrm{TeV}$ (leptogenesis through $y_{v}$ is irrelevant at such low scales, barring resonances). Taking $\epsilon=0.1$ and the $U(1)_{F}$ charges $q_{N 1}=7$,

\footnotetext{
8 The other way around, one could also enhance the hierarchy among the $M_{i}$ relatively to the hierarchy among the columns of $y_{v}$ and $y_{R}$, by introducing a (family-dependent) lepton number $U(1)_{L}$, that is conserved in the Yukawa couplings and broken by two units in the Majorana mass term. We will not need such a symmetry in the following.
} 
$q_{N 2}=q_{N 3}=2, q_{R \tau}=0 \geq q_{R \mu}, q_{R e}, q_{L \tau}=2 \geq q_{L \mu}, q_{L e}$, we can reproduce the correct size of the $M_{N}, y_{R}$ and $y_{\nu}$ entries, except for the second column of $y_{R}$, that must be further suppressed by $\epsilon_{R 2} \lesssim 10^{-3}$, and the first column of $y_{\nu}$, to be further suppressed by $\epsilon_{v 1} \lesssim 10^{-4}$. As discussed above, $\epsilon_{R 2}\left(\epsilon_{v 1}\right)$ can be associated to the breaking of $N_{2}$-parity $\left(N_{1^{-}}\right.$ parity). Note that here $M_{2,3} \sim \epsilon^{4} \Lambda_{L}$, with a lepton number violation scale $\Lambda_{L} \sim 10^{9} \mathrm{GeV}$.

In the case of leptogenesis from $N$-oscillations coupled to the SM through $y_{R}$, one requires for $i=2,3, M_{i}<100$ $\mathrm{GeV},\left|\left(y_{R}\right)_{\alpha i}\right| M_{i} \lesssim 10^{-5} \mathrm{GeV}$, as well as $\left|\left(y_{R}\right)_{\alpha i}\right|>10^{-7}$ for some flavour $\alpha$ and $<10^{-7}$ for some different flavour $\beta$. For definiteness, let us take $M_{1} \sim 10 \mathrm{keV}, M_{2,3} \sim 10 \mathrm{GeV}$ and $M_{\delta} \sim \mathrm{TeV}$. Then, one can reproduce the correct size of the $M_{N}$ and $y_{R}$ entries taking $\epsilon=0.1$ and $U(1)_{F}$ charges $q_{N 1}=8, q_{N 2}=q_{N 3}=5, q_{R \tau}=1, q_{R \mu}=2$ and $q_{R e}=3$, and charged lepton masses require $q_{L \tau}=1 \geq q_{L \mu}, q_{L e}$. Coming to the structure of $y_{v}$, one needs an extra source of suppression for $\left(y_{\nu}\right)_{\alpha 1}$, by a factor $\epsilon_{\nu 1} \lesssim 10^{-3}$, to avoid $N_{1}$-overproduction from active-sterile mixing; as before, $\epsilon_{v 1}$ can be understood as the breaking parameter of $N_{1}$-parity. We note that, for the chosen values of parameters, both $y_{R}$ and $y_{v}$ couplings to $N_{2,3}$ are relevant for leptogenesis. Here lepton number is broken at the scale $\Lambda_{L} \sim M_{2,3} / \epsilon^{10} \sim 10^{11}$ GeV.

In summary, the regions of parameters where our scenario is effective require a well-defined hierarchy of masses and couplings, that point to specific but relatively natural flavour structures, with no need of extreme tuning of parameters.

\section{Conclusions}

We have shown that, when the SM is extended with three sterile neutrinos $N_{i}$ and a charged scalar $\delta^{+}$, baryogenesis via leptogenesis and the production of dark matter can both be mediated by $\delta^{+}$.

The dark matter candidate in this scenario is the lightest sterile neutrino $N_{1}$, with a mass $M_{1}$ larger than about a few keVs. Such sterile neutrinos are produced by the decays of $\delta^{+}$while it is in thermal equilibrium in the early Universe. We showed that this novel mechanism, which is an example of freeze-in dark matter production, can account for the observed dark matter density. Moreover, since the resulting relic density does not depend on the active-sterile mixing angle $\theta_{1}$, the constraints from X-ray data are alleviated. The recent hint of a signal at $3.5 \mathrm{keV}$ can be easily explained by $N_{1}$-decays, for $\theta_{1} \sim 3 \times 10^{-6}$. Alternatively, for a vanishingly small $\theta_{1}$, the dark matter mass $M_{1}$ can be raised to the $\mathrm{MeV}$ scale, or beyond.

Leptogenesis can be realized through the coupling $y_{R}$ between $\delta^{+}$and $N_{2,3}$ in two different ways, either via $N$ decays or via $N$-oscillations. Both mechanisms are well- known in the context of the SM plus sterile neutrinos only, as they can both proceed through the neutrino Yukawa coupling $y_{v}$. The latter is constrained by light neutrino masses, restricting significantly the allowed parameter space and the testability of leptogenesis. In contrast, in our scenario $y_{R}$ is not involved in the neutrino mass generation. As a consequence, the $N$-decay mechanism is successful for $M_{2,3}$ as small as a few TeVs, with no need of a resonant enhancement. The parameter window for $N$-oscillation leptogenesis is similar when one employs the coupling matrix $y_{R}$ instead of $y_{v}$ : one needs roughly $M_{2,3} \sim \mathrm{GeV}$ and $y_{R}$-entries smaller than $10^{-6}$. However, $y_{R}$ introduces additional sources of $C P$ violation, and moreover it is not constrained neither by light neutrino masses nor by nucleosynthesis. Thus one can largely relieve the fine-tuning of parameters necessary in the case with $y_{v}$ only.

Neutrino masses are generated by a low scale seesaw mechanism involving the two heavier singlets, $N_{2,3}$, the $N_{1}$ contribution being negligible. In this way one can accommodate the current data on neutrino masses and mixing angles, with the prediction of an almost massless lightest neutrino. At colliders such as the LHC, this scenario can be probed and constrained via the direct production of $\delta^{+}$. This minimal extension of the SM thus provides a simple and testable scenario to explain neutrino masses, the dark matter, and the baryon asymmetry of the Universe.

Acknowledgments MF thanks F. Bezrukov, M. Drewes, T. Hambye, K. Jedamzik, P. Pralavorio and J. Racker for useful discussions. MF is partially supported by the FP7 European ITN project "Invisibles" (PITN- GA-2011-289442-INVISIBLES), and by the OCEVU Labex (ANR-11-LABX-0060) funded by the "Investissements d'Avenir" French government program managed by the ANR. C.Y. is partially supported by the "Helmholtz Alliance for Astroparticle Physics HAP" funded by the Initiative and Networking Fund of the Helmholtz Association.

Open Access This article is distributed under the terms of the Creative Commons Attribution License which permits any use, distribution, and reproduction in any medium, provided the original author(s) and the source are credited.

Funded by $\mathrm{SCOAP}^{3}$ / License Version CC BY 4.0.

\section{References}

1. S. Dodelson, L.M. Widrow, Sterile-neutrinos as dark matter. Phys. Rev. Lett. 72, 17-20 (1994). hep-ph/9303287

2. M. Fukugita, T. Yanagida, Baryogenesis without grand unification. Phys. Lett. B 174, 45 (1986)

3. K. Akhmedov Evgeny, V.A. Rubakov, A. Yu. Smirnov. Baryogenesis via neutrino oscillations. Phys. Rev. Lett. 81, 1359-1362 (1998). hep-ph/9803255

4. T. Asaka, M. Shaposhnikov, The nuMSM, dark matter and baryon asymmetry of the universe. Phys. Lett. B 620, 17-26 (2005). hep-ph/0505013 
5. T. Asaka, S. Blanchet, M. Shaposhnikov, The nuMSM, dark matter and neutrino masses. Phys. Lett. B 631, 151-156 (2005). hep-ph/0503065

6. L. Canetti, M. Drewes, T. Frossard, M. Shaposhnikov, Dark matter, baryogenesis and neutrino oscillations from right handed neutrinos. Phys. Rev. D 87, 093006 (2013). arXiv:1208.4607

7. K. Abazajian, S.M. Koushiappas, Constraints on sterile neutrino dark matter. Phys. Rev. D 74, 023527 (2006). astro-ph/0605271

8. A. Boyarsky, D. Iakubovskyi, O. Ruchayskiy, V. Savchenko, Constraints on decaying dark matter from XMM-Newton observations of M31. Mon. Not. R. Astron. Soc. 387, 1361 (2008). arXiv:0709.2301

9. S. Horiuchi, P.J. Humphrey, J. Onorbe, K.N. Abazajian, M. Kaplinghat, et al., Sterile neutrino dark matter bounds from galaxies of the Local Group. Phys. Rev. D 89, 025017 (2014). arXiv: 1311.0282

10. A. Boyarsky, J. Lesgourgues, O. Ruchayskiy, M. Viel, Lyman-alpha constraints on warm and on warm-plus-cold dark matter models. JCAP 0905, 012 (2009). arXiv:0812.0010

11. M. Viel, J. Lesgourgues, M.G. Haehnelt, S. Matarrese, A. Riotto, Can sterile neutrinos be ruled out as warm dark matter candidates? Phys. Rev. Lett. 97, 071301 (2006). astro-ph/0605706

12. M. Viel, G.D. Becker, J.S. Bolton, M.G. Haehnelt, Warm dark matter as a solution to the small scale crisis: new constraints from high redshift Lyman-alpha forest data. Phys. Rev. D 88(4), id., 043502 (2013). arXiv:1306.2314

13. X.-D. Shi, G.M. Fuller, A new dark matter candidate: nonthermal sterile neutrinos. Phys. Rev. Lett. 82, 2832-2835 (1999). astro-ph/9810076

14. M. Laine, M. Shaposhnikov, Sterile neutrino dark matter as a consequence of nuMSM-induced lepton asymmetry. JCAP 0806, 031 (2008). arXiv:0804.4543

15. M. Shaposhnikov, The nuMSM, leptonic asymmetries, and properties of singlet fermions. JHEP 0808, 008 (2008). arXiv:0804.4542

16. B. Shuve, I. Yavin, Baryogenesis through neutrino oscillations: a unified perspective. Phys. Rev. D 89, 075014 (2014). arXiv: 1401.2459

17. M. Shaposhnikov, I. Tkachev, The nuMSM, inflation, and dark matter. Phys. Lett. B 639, 414-417 (2006). hep-ph/0604236

18. F. Bezrukov, D. Gorbunov, Light inflaton Hunter's Guide. JHEP 1005, 010 (2010). arXiv:0912.0390

19. F. Bezrukov, D. Gorbunov, Relic gravity waves and $7 \mathrm{keV}$ dark matter from a GeV scale inflaton (2014). arXiv:1403.4638

20. F. Bezrukov, H. Hettmansperger, M. Lindner, keV sterile neutrino dark matter in gauge extensions of the standard model. Phys. Rev. D 81, 085032 (2010). arXiv:0912.4415

21. A. Kusenko, Sterile neutrinos, dark matter, and the pulsar velocities in models with a Higgs singlet. Phys. Rev. Lett. 97, 241301 (2006). hep-ph/0609081

22. A. Merle, V. Niro, D. Schmidt, New production mechanism for keV sterile neutrino dark matter by decays of frozen-in scalars. JCAP 1403, 028 (2014). arXiv:1306.3996

23. M. Frigerio, T. Hambye, E. Ma, Right-handed sector leptogenesis. JCAP 0609, 009 (2006). hep-ph/0603123

24. T. Asaka, M. Laine, M. Shaposhnikov, Lightest sterile neutrino abundance within the nuMSM. JHEP 0701, 091 (2007). hep-ph/0612182

25. A. Roy, M. Shaposhnikov, Resonant production of the sterile neutrino dark matter and fine-tunings in the [nu]MSM. Phys. Rev. D 82, 056014 (2010). arXiv: 1006.4008

26. L.J. Hall, K. Jedamzik, J. March-Russell, S.M. West, Freezein production of FIMP dark matter. JHEP 1003, 080 (2010). arXiv:0911.1120

27. M. Klasen, C.E. Yaguna, Warm and cold fermionic dark matter via freeze-in. JCAP 1311, 039 (2013). arXiv:1309.2777
28. E. Molinaro, C.E. Yaguna, O. Zapata, FIMP realization of the scotogenic model. JCAP 1407, 015 (2014). arXiv: 1405.1259

29. K. Petraki, A. Kusenko, Dark-matter sterile neutrinos in models with a gauge singlet in the Higgs sector. Phys. Rev. D 77, 065014 (2008). arXiv:0711.4646

30. A. Kusenko, Sterile neutrinos: the dark side of the light fermions. Phys. Rept. 481, 1-28 (2009). arXiv:0906.2968

31. K. Petraki, Small-scale structure formation properties of chilled sterile neutrinos as dark matter. Phys. Rev. D 77, 105004 (2008). arXiv:0801.3470

32. A. Kusenko, L.J. Rosenberg, Working Group Report: non-WIMP dark matter 1310, 8642 (2013)

33. Sacha Davidson, Alejandro Ibarra, A Lower bound on the righthanded neutrino mass from leptogenesis. Phys. Lett. B 535, 25-32 (2002). hep-ph/0202239

34. G.F. Giudice, A. Notari, M. Raidal, A. Riotto, A. Strumia, Towards a complete theory of thermal leptogenesis in the SM and MSSM. Nucl. Phys. B 685, 89-149 (2004). hep-ph/0310123

35. Apostolos Pilaftsis, CP violation and baryogenesis due to heavy Majorana neutrinos. Phys. Rev. D 56, 5431-5451 (1997). hep-ph/9707235

36. J. Racker, Mass bounds for baryogenesis from particle decays and the inert doublet model. JCAP 1403, 025 (2014). arXiv:1308.1840

37. J. Racker, N. Rius, Helicitogenesis: WIMPy baryogenesis with sterile neutrinos and other realizations (2014). arXiv:1406.6105

38. B. Garbrecht, More viable parameter space for leptogenesis (2014). arXiv: 1401.3278

39. L. Canetti, M. Shaposhnikov, Baryon asymmetry of the universe in the NuMSM. JCAP 1009, 001 (2010). arXiv:1006.0133

40. D. Gorbunov, M. Shaposhnikov, How to find neutral leptons of the vMSM? JHEP 0710, 015 (2007). arXiv:0705.1729

41. W. Bonivento, A. Boyarsky, H. Dijkstra, U. Egede, M. Ferro-Luzzi et al., Proposal to search for heavy neutral leptons at the SPS (2013). arXiv: 1310.1762

42. L. Canetti, M. Drewes, B. Garbrecht, Lab-to-Genesis (2014). arXiv: 1404.7114

43. O. Ruchayskiy, A. Ivashko, Restrictions on the lifetime of sterile neutrinos from primordial nucleosynthesis. JCAP 1210, 014 (2012). arXiv:1202.2841

44. M. Drewes, B. Garbrecht, Leptogenesis from a GeV seesaw without mass degeneracy. JHEP 1303, 096 (2013). arXiv:1206.5537

45. K. Abazajian, G.M. Fuller, W.H. Tucker, Direct detection of warm dark matter in the X-ray. Astrophys. J. 562, 593-604 (2001). astro-ph/0106002

46. E. Bulbul, M. Markevitch, A. Foster, R.K. Smith, M. Loewenstein, et al., Detection of an unidentified emission line in the stacked X-ray spectrum of galaxy clusters. Astrophys. J. 789, 13 (2014). arXiv: 1402.2301

47. A. Boyarsky, O. Ruchayskiy, D. Iakubovskyi, J. Franse, An unidentified line in X-ray spectra of the Andromeda galaxy and Perseus galaxy cluster (2014). arXiv:1402.4119

48. A. Boyarsky, J. Franse, D. Iakubovskyi, O. Ruchayskiy, Checking the dark matter origin of $3.53 \mathrm{keV}$ line with the Milky Way center (2014). arXiv: 1408.2503

49. T.E. Jeltema, S. Profumo, Dark matter searches going bananas: the contribution of potassium (and chlorine) to the $3.5 \mathrm{keV}$ line (2014). arXiv:1408.1699

50. D. Malyshev, A. Neronov, D. Eckert, Constraints on $3.55 \mathrm{keV}$ line emission from stacked observations of dwarf spheroidal galaxies (2014). arXiv:1408.3531

51. A. Boyarsky, J. Franse, D. Iakubovskyi, O. Ruchayskiy, Comment on the paper dark matter searches going bananas: the contribution of potassium (and chlorine) to the $3.5 \mathrm{keV}$ line by T. Jeltema and S. Profumo (2014). arXiv: 1408.4388 
52. M. Muhlleitner, M. Spira, A note on doubly charged Higgs pair production at hadron colliders. Phys. Rev. D 68, 117701 (2003). hep-ph/0305288

53. Serguei Chatrchyan et al., Search for a light charged Higgs boson in top quark decays in $p p$ collisions at $\sqrt{s}=7 \mathrm{TeV}$. JHEP 1207, 143 (2012). arXiv: 1205.5736

54. Search for charged Higgs bosons in the $\tau+$ jets final state with pp collision data recorded at $\sqrt{s}=8 \mathrm{TeV}$ with the ATLAS experiment. Technical report ATLAS-CONF-2013-090, CERN, Geneva, Aug 2013

55. E. Del Nobile, R. Franceschini, D. Pappadopulo, A. Strumia, Minimal matter at the large hadron collider. Nucl. Phys. B 826, 217-234 (2010). arXiv:0908.1567

56. G. Aad et al., Search for direct production of charginos, neutralinos and sleptons in final states with two leptons and missing transverse momentum in $p p$ collisions at $\sqrt{s}=8 \mathrm{TeV}$ with the ATLAS detector. JHEP 1405, 071 (2014). arXiv: 1403.5294

57. V. Khachatryan et al., Searches for electroweak production of charginos, neutralinos, and sleptons decaying to leptons and $\mathrm{W}, \mathrm{Z}$, and Higgs bosons in pp collisions at $8 \mathrm{TeV}$ (2014). arXiv: 1405.7570
58. G. Aad et al., Search for the direct production of charginos, neutralinos and staus in final states with at least two hadronically decaying taus and missing transverse momentum in $p p$ collisions at $\sqrt{s}=8 \mathrm{TeV}$ with the ATLAS detector. JHEP 1410, 96 (2014). arXiv: 1407.0350

59. A. Merle, V. Niro, Deriving models for $\mathrm{keV}$ sterile neutrino dark matter with the Froggatt-Nielsen mechanism. JCAP 1107, 023 (2011). arXiv:1105.5136

60. J. Barry, W. Rodejohann, H. Zhang, Sterile neutrinos for warm dark matter and the reactor anomaly in flavor symmetry models. JCAP 1201, 052 (2012). arXiv: 1110.6382

61. M. Shaposhnikov, A possible symmetry of the nuMSM. Nucl. Phys. B 763, 49-59 (2007). hep-ph/0605047

62. M. Lindner, A. Merle, V. Niro, Soft $L_{e}-L_{\mu}-L_{\tau}$ flavour symmetry breaking and sterile neutrino $\mathrm{keV}$ dark matter. JCAP 1101, 034 (2011). arXiv: 1011.4950

63. A. Merle, keV neutrino model building. Int. J. Mod. Phys. D 22, 1330020 (2013). arXiv:1302.2625 